\title{
Rare Cellulite On Mucormycosis
}

\author{
Joumany Brahim Salem, Imane Tarib, Mahdi Khammaily, Sidi Dahi, Youssoufou Souley Abdoul \\ salam, Mohamed El Habib bahlou, Essaoudi Mohamed Amine, Rachid Zarrouk, Yassine Mouzari, \\ Fouad El Asri, Karim Reda, and Abdelbarre Oubaaz
}

\begin{abstract}
Mucormycosis is a serious fungal infection that is associated with high mortality, but is rarely reported in ophthalmology. It is a disease with various presentations, this is why it is important to think about it in unbalanced diabetic patients. We report the case of a 27 -year-old patient admitted for febrileke to acidosis decompensation of anorbito-facial cellulitis.
\end{abstract}

Index Terms - Diabetes, Mucormycosis, Orbital cellulitis, Sinusitis.

\section{INTRODUCTION}

Mucormycosis is a rare, rapidly spreading opportunistic fungal infection with an unspecific clinical and radiological presentation. It is responsible for a high mortality rate.

\section{PATIENTS AND OBSERVATIONS}

We report the case of a 27 years old patient, diabetic for 7 years on insulin, admitted to the emergency room for febrileke to acidosis decompensation with an intense headache and a left retro-orbital pain evolving for a week. Upon inspection, the patient was conscious but restless, with palpebral and jugal edema associated with an inferiorly dislocation of the nasal septum, a ptosis and left unilateral exophthalmos. Ophthalmological examination of the left eye noted visual acuity with negative light perception, complete oculomotor paralysis, fixe dilated pupil and at the fundus examination a diffuse retinal pallor with uninhabited vessels evoking an arterio-venous vascular occlusion (Figure 1). Examination of the right eye revealed no abnormalities (Figure 2). The rhino-sinus examination showed lysis of the nasal septum, palatal ulceration with blackish crusts. The neurological examination did not show any sensitive or motor deficit and the hemodynamic state was stable. The biological assessment revealed a hyperglycemia at 27.2 mmol / 1 with a glycated hemoglobin at $8.1 \%$ and a biological inflammatory syndrome. Orbito-cerebral computed tomography (CT) performed in the emergency department showed a pan-sinusitis complicated by ethmoid abscess and bone lyses associated with facial and orbital left retro-septal cellulitis with exophthalmos and intra-conical infiltration reaching the orbital apex and including the optic nerve up to the left optic canal (Figure 3). Orbito-cerebral magnetic resonance imaging (MRI) showed facial and orbital cellulitis, mucous thickening in the frame of the frontal and maxillary sinuses with filling of the ethmoid cells, the sphenoid and frontal sinuses; associated with optic nerve signal abnormalities consistent with optic neuritis
(Figure 4). Cerebral ANGIO-MRI revealed no signs of thrombosis of the cavernous sinus or abnormal arterial or venous sequences (Figure 5). Exploration under general anesthesia in the operating room revealed a lysis of the septum and the osteo-sino-nasal septum extended with blackish necrotic tissue, a lysis of the middle and lower horns and a left ethmoid bulge. The patient underwent debridement with pus removal and an ethmoidal and sphenoidal biopsy. The diagnosis of mucormycosis was made, in the context of pan sinusitis with blackish crust and necrosis of the nasal mucosa in an unbalanced diabetic patient. General antifungal treatment with Amphotericin B at a dose of $1 \mathrm{mg} / \mathrm{kg} /$ day was started combined with antibiotic therapy and intravenous insulin therapy. The direct examination of the sinus mycological samples found mycelial filaments. The biopsy showed sketches of necrosis and short unseptated mycelial filaments associated with spores (Figure 6). Mycological culture analysis identified the agent Rhizopus arrhizus. Multiple surgical nasal sinus debridements, endo-nasally under local anesthesia were repeated when ever deemed necessary. The subsequent course was favorable with regression of pan-sinusitis and exophthalmos but the blindness was definitive. Amphotericin B was maintained for 3 months with monitoring of renal and hepatic function throughout the duration of treatment.



Fig 1: Left Eye retinal necrosis with uninhabited vessels 


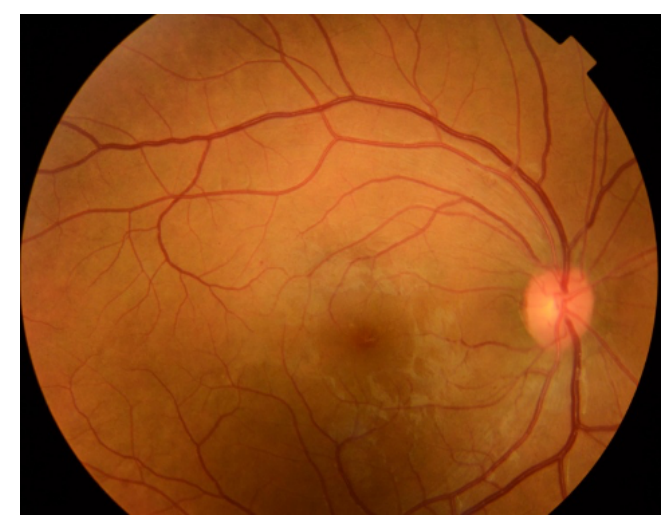

Fig 2: Normal right eye.



Fig 3: Orbito-cerebral CT retro-septal orbital cellulitis with infiltration of the left optic nerve.

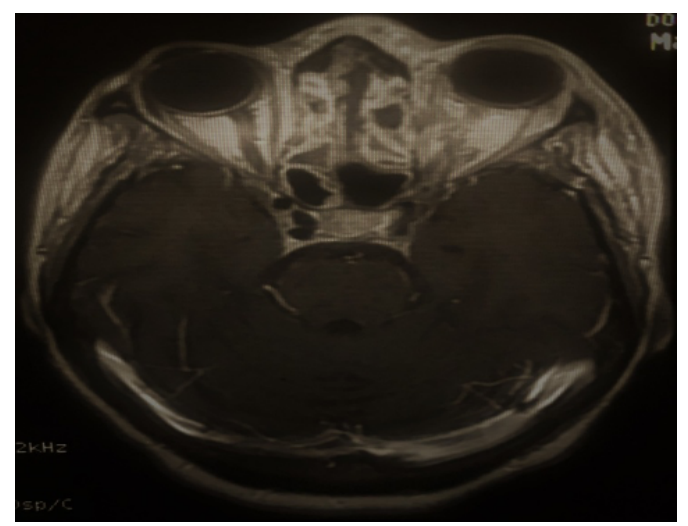

Fig 4: Orbito-cerebral MRI showing filling of sinus and optic nerve signal abnormality.



Fig 5: Brain ANGIO-MRI showing absence of abnormal arterial or venous sequences.

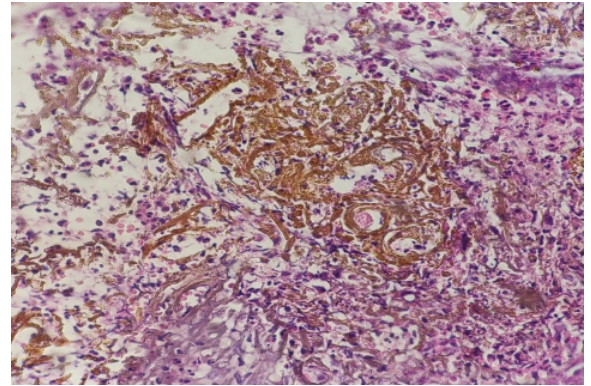

Fig 6: (HE x40): Conjunctival squamous epithelium showing rough necrosis and thickmycelial filaments.

\section{DISCUSSION}

Mucormycosisis an angio-invasive fungal infection, associated with high morbidity and mortality but it is poorly understood in ophthalmology because it is rarely reported; this may be due to the fact that these are patients who are mainly treated in the intensive care unit. It is due to an overgrowth in the tissues of a mucous class Zygomycete, the main germ of which is Rhizopus [1]. These usually saprophytic microorganisms become pathogenic in diabetic patients especially those with ketoacidosic decompensation under the effect of hyperglycemia and the reduction of the phagocytic function of neutrophils, thus promoting the growth of fungi [2]. Blood vessels, causing mucothrombosis that is the source of tissue necrosis, which characterizes the anatomopathological results found in biopsies [3]. The infection begins in the nasal or oral mucosa to spread to the ethmoid and maxillary sinuses, then the frontal and sphenoid sinuses. Orbital extension is either by contiguity or by the peri-vascular or peri-neural route [4]. It results clinically in ophthalmoplegia, exophthalmos and inflammatory ptosis [2] [5]. Ocular paralysis is secondary to either paralysis of the cranial pairs or direct damage to the oculomotor muscles [3,5]. Extension of the infection to the orbital apex results in optic neuritis causing blindness [2]. The diagnosis is evoked before the association of pansinusitis with blackish crusts or bedsores in the orbito-nasal region or the floor of the mouth [5]. The confirmation of diagnosis depends enormously on the anatomo pathological examination findings, mycelial filaments, thick, short, not septate, having ramifications at right angles corresponding to mucores. Culture on sabouraud medium makes it possible to identify the species and carry out the antibiogram [6]; but these cultures are not always positive, because the fungal elements are rare and distributed in a heterogeneous way in the tissue fragments [7]. The treatment of naso-orbital mucormycosis is based on 3 components: the balancing of risk factors, antibiotic therapy and surgical debridement [2] [5] [6]. Antibiotic therapy is based on amphotericin B intravenously at a dose of $1 \mathrm{mg} / \mathrm{kg} /$ day maintained for 3 months. The liposomal form $(10 \mathrm{mg} / \mathrm{kg} /$ day $)$ provides a better response with fewer side effects [8]. Surgical debridement of the foci of necrosis must be carried out early and guided by the extemporaneous examination [9]. The functional and vital prognosis of this condition is bad with a mortality rate of 20 to $50 \%$ of cases [10]. Survival depends on the rapid response time ( $76 \%$ for treatment before 7 days and $40 \%$ after two weeks) and on the combination of surgical debridement with antibiotic therapy [3] [10]. 


\section{CONCLUSION}

Mucormycosis is a severe, emerging and fatal infection requiring multidisciplinary management. It is a disease with various presentations, hence the importance of keeping it in mind when confronting unbalanced diabetic or immunocompromised patients, in the presence of blackish crust or foci of necrosis on endoscopic examination. The importance being an early start of the medico-surgical treatment to improve life prognosis and give patients a higher chance.

\section{REFERENCES}

[1] Jeong W, Keighley C, Wolfe R, Lee WL, Slavin MA, Kong DCM, et al. The epidemiology and clinical manifestations of mucormycosis: a systematic review and meta-analysis of case reports. Clin Microbio Infect Off Publ Eur Soc Clin Microbiol Infect Dis. 2019;25(1):2634.

[2] Bhansali A, Bhadada S, Sharma A, Suresh V, Gupta A, Singh P, et al. Presentation and outcome of rhino-orbital-cerebral mucormycosis in patients with diabetes. Postgrad Med J. 2004;80(949):670-674.

[3] Wali U, Balkhair A, Al-Mujaini A. Cerebro-rhino orbital mucormycosis: An update. J Infect Public Health. 2012;5(2):116126.

[4] Narayanan S, Panarkandy G, Subramaniam G, Radhakrishnan C, Thulaseedharan N, Manikath N, et al. The "black evil" affecting patients with diabetes: a case of rhino orbito cerebral mucormycosis causing Garcin syndrome. Infect Drug Resist. 2017;10:103-108.

[5] Mbarek C, Zribi S, Khamassi K, Hariga I, Ouni H, Ben Amor M, et al. Rhinocerebral mucormycosis: five cases and a literature review. B-ENT. 2011;7(3):189-193.

[6] Jain S, Kumar S, Kaushal A. Rhinocerebral mucormycosis with isolated sixth nerve palsy in an immunocompetent patient. Med J Malaysia. 2011;66(4):376-378.

[7] Gaillard T, Crémades A, Cathelinaud O, Crémades S, Brisou P, Terrier J-P, et al. Diagnostic d'une mucormycose. 2005;3.

[8] Ogawa T, Takezawa K, Tojima I, Shibayama M, Kouzaki H, Ishida $\mathrm{M}$, et al. Successful treatment of rhino-orbital mucormycosis by a new combination therapy with liposomal amphotericin B and micafungin. Auris Nasus Larynx. 2012;39(2):224-228.

[9] Saedi B, Sadeghi M, Seilani P. Endoscopic management of rhinocerebral mucormycosis with topical and intravenous amphotericin B. J Laryngol Otol. 2011;125(8):807-810.

[10] Chander J, Kaur J, Gulati N, Arora V, Nagarkar N, Sood S, et al. Sudden vision loss caused by rhino-orbital zygomycosis in diabetic patients: case series. Mycoses. 2011;54(4):e228-232.

\section{Joumany Brahim Salem}

Corresponding e-mail: joumanybss@gmail.com

Institution: Department of Ophthalmology, Mohammed V Military Instructional Hospital, Faculty of Medicine and Pharmacy,

Mohammed V University, Rabat, Morocco.

\section{Imane Tarib}

e-mail: amaitarib@gmail.com

Institution: Department of Ophthalmology, Mohammed V Military Instructional Hospital, Faculty of Medicine and Pharmacy,

Mohammed V University, Rabat, Morocco.

\section{Mahdi Khammaily}

e-mail: mehdi.khamaily@gmail.com

Institution: Ophthalmology Department, Mohammed Military Instruction

Hospital V, Faculty of Medicine and Pharmacy,

Mohammed V University, Rabat, Morocco.

\section{Sidi Dahi}

e-mail: dahisidi2014@gmail.com

Institution: Ophthalmology Department, Mohammed V Military Instruction

Hospital, Faculty of Medicine and Pharmacy,

Mohammed V University, Rabat, Morocco.
Mohamed EI Habib bahlou

Institution: Oto Rhino Laryngology Service, Military Hospital Mohammed $\mathrm{V}$, Faculty of Medicine and Pharmacy,

Mohammed University V, Rabat, Morocco.

Essaoudi Mohamed Amine

Institution: Department of anatomopathology, Military Hospital Mohammed V, Faculty of Medicine and Pharmacy,

Mohammed University V, Rabat, Morocco.

\section{Yassine Mouzari}

e-mail: ys.mouzari@gmail.com

Institution: Department of Ophthalmology, Military Hospital of Instruction

Mohammed V, Faculty of Medicine and Pharmacy,

Mohammed V University, Rabat, Morocco.

\section{Fouad El Asri}

e-mail: elasri_76@hotmail.com

Institution: Ophthalmology Service, Mohammed V Military Instructional Hospital, Faculty of Medicine and Pharmacy,

Mohammed V University, Rabat, Morocco.

\section{Karim Reda}

e-mail: mkarimreda@gmail.com

Institution: Ophthalmology Department, Mohammed V Military Instruction Hospital, Faculty of Medicine and Pharmacy,

Mohammed V University, Rabat, Morocco.

\section{Abdelbarre Oubaaz}

e-mail: aoubaaz@gmail.com

Institution: Ophthalmology Service, Military Instruction Hospital

Mohammed V, Faculty of Medicine and Pharmacy,

Mohammed V University, Rabat, Morocco. 